\section{Endoscopic ultrasound-guided rescue of an uncovered self-expanding metallic stent causing biliary obstruction}

Removal of a malpositioned uncovered Wallstent (Boston Scientific, Natick, Massachusetts, USA) can be extremely difficult, time-consuming, and associated with the occurrence of complications [1]. Stent extraction using standard polypectomy snare or rat-tooth forceps is often unsuccessful [1]. Alternatively stent removal can be accomplished by piecemeal extraction of individual stent filaments [1-4]. We present a case in which drainage of the biliary system, obstructed by a Wallstent wedged to the hilum, was achieved by endoscopic ultrasound (EUS)-guided puncture of the left intrahepatic bile duct followed by rendezvous endoscopic retrograde cholangiography (ERC).

A 57-year-old man with metastatic cholangiocarcinoma, who had undergone a previous ERC with placement of an uncovered biliary Wallstent, was referred to us for evaluation of persistent jaundice (bilirubin $58 \mathrm{mg} / \mathrm{dl}$ ). A repeat ERC showed complete occlusion of the Wallstent, with some contrast spilling into the right biliary system and complete obstruction of the left system (Figure $\mathbf{1} \mathbf{a}$ ).

After multiple unsuccessful attempts at advancing a guide wire through the stent, the duodenoscope was exchanged for a linear-array echo endoscope (Olympus America, Melville, New York, USA), that was used to identify the dilated bile ducts within the left hepatic lobe and to puncture them using a 19-gauge needle. After bile had been aspirated and contrast injected to opacify the biliary system, a 0.035-inch Jagwire (Boston Scientific) was inserted through the needle and advanced antegradely across the mesh of the Wallstent, the hilar stricture, and into the duodenum (Figure $\mathbf{1} \mathbf{b}$ ). A rendezvous ERC was finally performed, with placement of a $6 \mathrm{~cm}$ long, $6 \mathrm{~mm}$ wide Zilver stent (Wilson-Cook Medical, WinstonSalem, North Carolina, USA) through the meshes of the Wallstent (Figure $\mathbf{1}$ c). Serum bilirubin levels were completely normalized 10 weeks after the procedure.
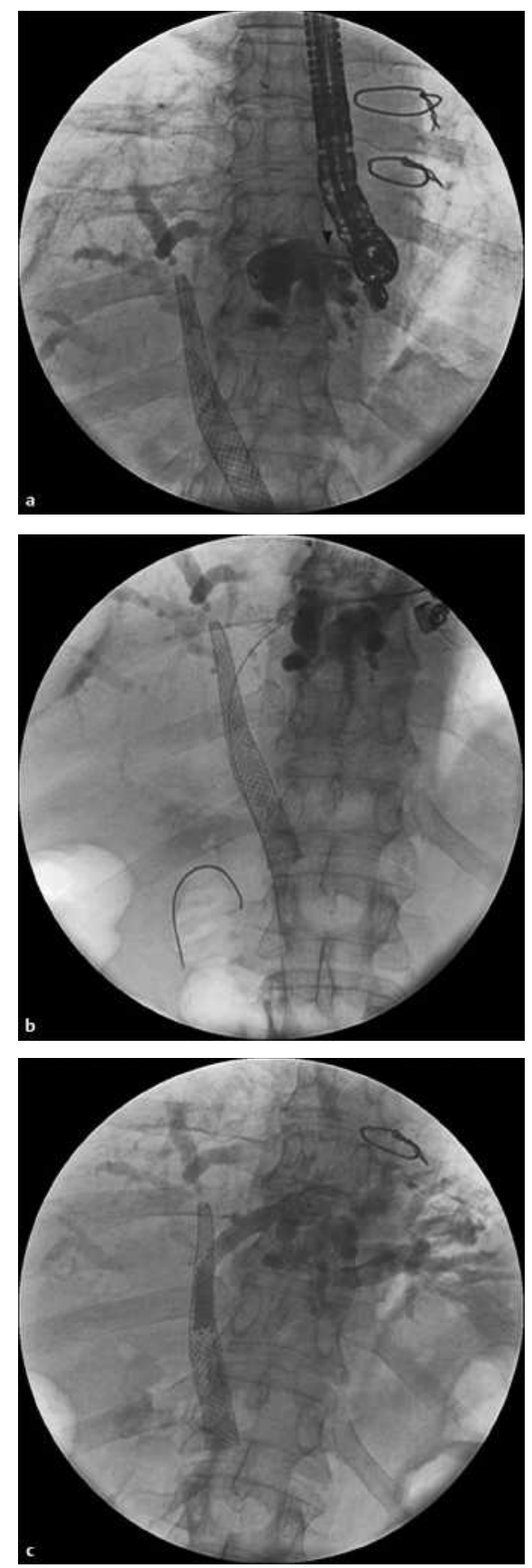

Figure 1 Fluoroscopic views of the procedure. a Endoscopic ultrasound (EUS)-guided transhepatic contrast injection with a 19gauge needle (arrow), and the malpositioned uncovered Wallstent with its proximal end wedged into the hilum. $\mathbf{b}$ The guide wire was inserted from the left hepatic duct through the stent and into the duodenum. c The Zilver stent was placed through the mesh of the Wallstent.
We have reported here an illustrative case of EUS-guided rendezvous biliary drainage; this approach can be considered as a valid alternative to the percutaneous transhepatic route when a malpositioned SEMS, not amenable to endoscopic removal, is the cause of obstruction.

Endoscopy_UCTN_Code_TTT_1AR_2AZ

Endoscopy_UCTN_Code_TTT_1AS_2AD

Endoscopy_UCTN_Code_CPL_1AK_2AD

\section{A. Larghi, I. Waxman}

Section of Endoscopy and Therapeutics and the Cancer Research Center, The University of Chicago, Chicago, Illinois, USA.

\section{References}

${ }^{1}$ Kahaleh M, Tokar J, Le T et al. Removal of self-expandable metallic Wallstents. Gastrointest Endosc 2004; 60: 640-644

${ }^{2}$ Ahmed A, Keeffe EB, Imperial JC. A novel technique for endoscopic removal of expandable biliary Wallstent. Gastrointest Endosc 1999; 50: 279-281

${ }^{3}$ Egan LJ, Baron TH. Endoscopic removal of an embedded biliary Wallstent by piecemeal extraction. Endoscopy 2000; 32: 492-494

${ }^{4}$ Levy MJ, Wiersema MJ. Endoscopic removal of a biliary Wallstent with a suture-cutting device in a patient with primary pancreatic lymphoma. Endoscopy 2002; 34: 835-837

\section{Corresponding author}

\section{Waxman, M.D.}

The University of Chicago

Section of Gastroenterology

5758 S. Maryland Avenue, MC 9028

Chicago, Illinois 60637

Fax: +1-773-834-7209

E-mail: iwaxman@medicine.bsd. uchicago.edu 